\title{
Contrast Enhancement of Ultrasound Image of Knee Joint Cartilage by Using Multipurpose Beta Optimized Recursive Bi-Histogram Equalization Method
}

\author{
Md Belayet Hossain ${ }^{\text {a, }}$, Belinda Pingguan-Murphy ${ }^{\text {a }}$, Yan Chai Hum ${ }^{\text {b }}$, Khin Wee Lai ${ }^{{ }^{*}}$ \\ ${ }^{a}$ Biomedical Engineering Department, Faculty of Engineering, University Malaya, Kuala Lumpur, 50603, Malaysia \\ ${ }^{\mathrm{b}}$ Advanced Informatics Architecture Development, MIMOS Berhad, Technology Park Malaysia, 57000, Kuala Lumpur \\ *Corresponding Author: lai.khinwee@um.edu.my
}

\begin{abstract}
Multipurpose Beta Optimized Recursive Bi-histogram Equalization (MBORBHE) method has been proposed in this article for contrast enhancement of US image. Histogram equalization (HE) is very popular for contrast enhancement. But conventional HE methods do not consider brightness and detail preservation simultaneously during contrast enhancement of US image. In case of our proposed method brightness and detail preservation will be considered simultaneously during contrast enhancement of US image. For achieving this goal, a final objective function will be constructed by considering three individual objective functions named as preservation of brightness score (PBS), Optimum contrast score (OCS) and Preservation of detail score (PDS). The final objective function will be used to find out the optimum separating point for segmenting the histogram of the input US image for which brightness and detail preservation will be obtained during optimum contrast enhancement of the US image. US image of knee joint cartilage will be used for the performance evaluation of the proposed method. Qualitative analysis of the proposed method will be performed by using human visual perception. Different performance metrics named as Signal to noise ratio (SNR), Structure Similarity Index Measurement (SSIM), Entropy and shift of mean brightness will be bring into account for quantitative analysis of the proposed method. As there is a significant change of the shape of cartilage with the progression of knee Osteoarthritis (OA), so with the contrast enhanced US image of knee joint cartilage, it will be possible to detect early knee OA.
\end{abstract}

Keywords: ultrasound, osteoarthritis, contrast, functions.

\section{Introduction}

Osteoarthritis (OA) is most common form of arthritis which creates, initially, painful experience to the patients and finally, leads to joint effusion. In the world, more than $80 \%$ of the populations have radiographic evidence of OA by age 65 . When water content of a cartilage increases due to natural elderly, protein level of the cartilage degenerates. As a result, cartilage begins to degenerate by flaking or forming tiny crevasses. Cartilage and Synovial fluid drop their natural properties to perform as cushion and lubricant in the joints. MRI is superior to provide radiographic evidence of early OA.

However, MRI is expensive and not suitable to the implanted patients. X-rays gives ionizing radiation as a result of high risk of getting cancer. CT scan is not suitable for detecting inflammation or infection. It also emits higher level of radiation. Ultrasound is beneficial in terms of costs and availability. It is also non-invasive, non-ionizing radiation, cost effective, portable, no need for special environment and applicable to any patient. Real time imaging is also possible by using US medical imaging system. However it has some limitations such as its contrast ratio is very low and affected by the speckle noise. Its resolution is also poor compared to MRI image; moreover, its efficiency is much dependent on operator skill. Cartilage loss is the main feature of the knee OA. By using MRI it is possible to directly visualize the articular hyaline cartilage. Assessments of cartilage morphology from knee MRI are emerging as promising measures for monitoring OA disease progression [1]. However, Ultrasound has potentiality to be very precise tools to diagnosis early $\mathrm{OA}$, if its image can be improve by image processing. Therefore, the hypothesis is- 
by image processing, Ultrasound can be utilized as a perfect tool to detect early OA. The main objective of this study is to improve US image to overcome the limitation of low contrast which will be helpful for the early detection of knee OA. The outcome of the study will be a novel technique of getting information on early knee OA by using US Imaging. Histogram equalization (HE) is a very popular technique for enhancing contrast of images [2]. Low contrast ration of US image can be enhanced by using Histogram Equalization (HE). But existing HE methods only focus on either brightness or detail preservation. For that reason we have to improve the conventional HE method to overcome its existing limitations. In case of conventional HE method, selecting the appropriate separating point for segmenting the histogram is the main challenge. By using the proposed method we will be able to select the separating point for segmenting histogram so that at the same time brightness and detail preservation are maintained during contrast enhancement of the US image.

\section{Different Histogram Equalization (HE) Methods}

All The basic principle of HE or GHE (global histogram equalization) is to map the gray levels based on probability distribution of the input image's gray levels. By using image's cumulative density function, it stretches the dynamic range of the image, so it will improve the contrast of the image. HE has been successfully applied in various fields such as radar image processing and medical image processing [ $[\underline{3}, \underline{4}]$. In most image processing packages such as Adobe Photoshop []], Lispix [] ] and National Institute of Health Image [7] this popular HE method is also available. HE will flat the histogram of the input image where the whole gray level has uniform probability density. Here probability density of the gray level, $x \in\left\{x_{0}, x_{1} \ldots \ldots x_{L-1}\right\}$ is denoted by $P\left(X_{K}\right)$ and $L$ indicates the total number of gray levels of the image. For a given image $X$, the probability density function is defined as

$$
p\left(X_{K}\right)=\frac{n^{k}}{n}
$$

For $k=0,1, \ldots, L-1$, where $n^{k}$ denotes the number of time, that the level of $x_{k}$ appears in the input image $X$. Total number of pixel in the input image is $n$. A plot of $n^{k}$ vs $x_{k}$ is known as the histogram of $X$. Based on the probability density function, the cumulative density function is defined as

$$
c(x)=\sum_{j=0}^{k} p\left(x_{j}\right)
$$

Here, $x_{k}=X$, for $k=0,1, \ldots, L-1$. By definition c $\left(X_{L-1}\right)$ $=1$. Histogram equalization is then used to map the image into the entire dynamic range $\left[X_{0}-X_{N-1}\right]$. It is done by using the cumulative density function, shown as the following equation

$$
f(X)=X_{0}+\left(X_{N-1}-X_{0}\right) * c(x)
$$

which flattens the histogram of an image and causes a significant change in the brightness. The equation of the output image of the GHE is $Y=\{Y(i, j)\}$, which can be expressed as

$$
Y=f(x)=\{f X(i, j) \mid \forall X(i, j) \in X
$$

However, the mean shift remains the well-known problem for the conventional HE method, which indicates that the mean brightness of the input and output image change significantly. This method also introduce undesirable artifacts. For these disadvantages, HE is rarely used on scanning electron microscope (SEM) images. In case of HE, It has been observed that the middle gray level is always the mean brightness of the histogram-equalized image regardless of the input image as shown by the following equation.

$$
E(Y)=X_{G}=1 / 2\left(X_{0}+X_{L-1}\right)
$$

Here, $Y$ denotes the output image, mean is indicated by $E($.$) and X_{G}$ denote the middle of the gray level. This property hamper the preservation of brightness of the input image.

To overcome this limitations kin []ㅡ proposed Brightness Preserving Bi-Histogram Equalization (BBHE). Firstly this method will segment the input image into two sub-images based on the mean of the input image $x_{m} \in x_{0}, x_{1}, \ldots . x_{L-1}$. After that conventional HE method is applied on each sub-image independently. The resultant image will be obtained by composing sub-images into one image. From experimental and mathematical analysis it has been observed that this method can preserve brightness of the input image at a certain extent. In case of Dualistic sub-image histogram equalization (DSIHE) [9] the histogram of the input image is segmented by using median instead of mean. Then conventional HE is applied in each sub-image independently. Processed sub-image is composed into one image produce the resultant image. Recursive Mean Separate Histogram Equalization (RMSHE) [10] and Recursive sub-image histogram equalization (RSIHE) [11] are the recursive version of BBHE and RSIHE respectively. Minimum mean brightness error bio-histogram equalization (MMBEBHE) [12] uses each gray level for segmenting the histogram of the input image. Then it will determine for which gray level the 
Absolute Mean Brightness Error (AMBE) of input image and contrast enhanced image become minimum. Then this gray level will be used for segmenting the histogram of the input image.

\section{Materials and Methods}

Papers The basic idea of MBORBHE is to find out a separating point for segmenting the histogram of the input image for which the optimum value of preservation of brightness score, preservation of detail score and contrast enhancement score will be considered. In this method firstly the input image will be decomposed into two sub-images by using every possible separating point. Then global histogram equalization is applied in each sub-images independently. For measuring preservation of brightness, preservation of detail and optimum enhancement of contrast three defined objective functions will be used. By using weighted sum approach we will form a final objective function which will combine the three defined objective functions. Function output is computed by repeating each possible decomposition point for the bi-histogram equalization. By considering maximum value of objective functions resultant image was selected. Three objective functions named as preservation of brightness score (PBS), optimum contrast score (OCS) and preservation of detail score (PDS) are defined below.

\subsection{Preservation of Brightness Score (PBS) function}

For defining the preservation of Brightness score $(P B S)$ function, firstly it is needed to find out relative brightness difference between input and output (histogram equalized) image. For this the following two equations will be derived.

$$
\begin{aligned}
& \mu_{X}=\frac{1}{M N} \sum_{I=0}^{M} \sum_{J=0}^{N} I_{x}(i, j) \\
& \mu_{y}=\frac{1}{M N} \sum_{I=0}^{M} \sum_{J=0}^{N} I_{y}(i, j)
\end{aligned}
$$

Here, the input and output image mean is $\mu_{x}$ and $\mu_{y}$ respectively. The pixel intensity of input image at spatial location $(i, j)$ is indicated by $I_{x}(i, j)$ and pixel intensity of output image at spatial location $(i, j)$ is denoted by $I_{y}(i, j)$. $M$ and $N$ indicate the image dimensions.

The mean brightness difference can be described as $\mu_{y}-\mu_{x}$. But this difference is not suitable to be used due to two demerits: Firstly, there exists no standard bounding value which is important to compare with enhanced contrast and detail retention. Secondly, it lacks of flexibility in manipulating its expected behavior.
Hence, to resolve the first downside it is crucial to map brightness difference score onto the unit interval $[0,1]$, so that the value represents the degree of brightness deviation instead of the absolute value of brightness difference. This mapping is performed by using the normalization formula for relative brightness deviation as shown in below.

$$
N B\left(\mu_{y}, \mu_{x}\right)=\frac{\left|\mu_{y}-\mu_{x}\right|}{\mu_{x}+\mu_{y}+C}
$$

Here $\mathrm{c}$ is any arbitrary small constant used to assure computation stability in extreme cases. The numerical value of (8) ranges from 0 to 1 . As the output value of $N B$ (Normalized Brightness) function approaches unity, it implies that the degree of deviation in brightness between the input image and output image is high, and vice versa

It is needed to insert the output of (8) as input of another function for solving the second problem of manipulating the expected behaviour. The purpose of this function is to model the expected behaviour. For the sake of simplicity, this function is required to exhibit different behaviours or function output's shape by controlling only a few parameters in order to model the expert's opinion and knowledge conveniently. In this case, we opt for beta distribution function with the parameter $\alpha_{1}$ and $\beta_{1}$ and this function is termed as Preservation of Brightness Score (PBS)

$$
\begin{aligned}
& P B S=\frac{\Gamma\left(\alpha_{1}+\beta_{1}\right)}{\Gamma\left(\alpha_{1}\right)+\Gamma\left(\beta_{1}\right)}(1-N B)^{\beta_{1}-1} N B^{\left(\alpha_{1}-1\right)} \\
& \Gamma(x)=\int_{0}^{1} e^{-t} N B^{(X-1)} d(N B)
\end{aligned}
$$

Where $\Gamma($.$) is the Gamma function.$

Again, to make sure that the output of $P B S$ is of unit interval, it is normalized by using the maximum value of the function as the ratio of $P B S$ to the max value of $P B S$ over the range of $N B$, this normalized value of $P B S$ is termed as the normalized Preservation of Brightness Score, NPBS:

$$
N P B S=\frac{P B S}{\operatorname{argmax}(P B S)}
$$

The motivation of NPBS is to model the perception of human visual system. An expert in visual application can use it to adjust the optimum value of desired range of brightness difference. The output value of NPBS can be viewed also as the membership function of brightness deviation value. 'Smaller the brightness difference value better the output image' was the previous concept. In case of our proposed method NPBS will be used for giving emphasis on human visual perception. As $N P B S$ approaches 
unity, it implies that the resultant image is more preferable for human visual perception as well as optimum brightness preservation. The parameters of NPBS also determine the location of high values and low values of $N P B S$ and hence determine the preference of brightness deviation by the user.

By using $P B S$, it is possible to measure the difference of mean brightness of input and output image. However, the very small brightness difference that was found by the previous researches was not able to be found by using $N B$. So, human visual perception was not favourable in case of $N B$. For that reason, $P B S$ or $N P B S$ had been used. $N P B S$ is a normalized function defined as the ratio of $P B S$ and maximum value of $P B S$. $N B$ was mapped by using $N P B S$ to a new set of value ranges from 0 to 1 . Beta distribution has been used for mapping by using parameters $\alpha_{1}$ and $\beta_{1}$. $N P B S$ provided us some values that were used to measure the brightness preservation quantitatively. However, in case of traditional method, the smallest brightness difference indicates the superiority of the resultant image. Good or bad brightness preserving ability can be defined by using NPBS function with the help of $N B$.

\subsection{Optimum Contrast Score (OCS) function}

For defining the optimum contrast score (OCS) function, firstly normalized contrast $(N C)$ is defined. For this it is needed to find out normalized root mean square contrast of input image $\left(\sigma_{\mathrm{x}}\right)$ and output image $\left(\sigma_{\mathrm{y}}\right)$.

$$
\begin{aligned}
\sigma_{x} & =\sqrt{\frac{1}{M N}} \sum_{i=o}^{M-1} \sum_{j=0}^{N-1}\left(I_{X}(i, j)-\mu_{x}\right)^{2} \\
\sigma_{y} & =\sqrt{\frac{1}{M N}} \sum_{i=o}^{M-1} \sum_{j=0}^{N-1}\left(I_{y}(i, j)-\mu_{y}\right)^{2} \\
N C & =1-\frac{\left|\sigma_{y}-\sigma_{x}\right|}{\sigma_{x}+\sigma_{y}+c}
\end{aligned}
$$

Constant $\mathrm{c}$ is used for maintaining the function stability. The value of $N C$ ranges from 0 to 1 . By using beta distribution and parameter $\alpha_{2}$ and $\beta_{2}$ the equation of optimum contrast (OCS) score is defined

$$
O C S=\frac{\Gamma\left(\alpha_{2}+\beta_{2}\right)}{\Gamma\left(\alpha_{2}\right)+\Gamma\left(\beta_{2}\right)}(1-N C)^{\beta_{2}-1} N C^{\left(\alpha_{2}-1\right)}
$$

where

$\Gamma($.$) is the Gamma function.$

$$
\text { NOCS }=\frac{\text { OCS }}{\operatorname{argmax}(O C S)}
$$

The relation between $N C$ and NOCS are analogous to $N B$ and $N P B S$. As relatively high or low contrast is not preferable for the US image, the proposed method will find out the optimum contrast value by using NOCS.

\subsection{Preservation of Detail Score Function (PDS)}

For preservation of detail score function $(P D S)$, firstly we have to find out normalized detail $(N D)$ which is the simultaneous pixel mean intensity change between input image and output image.

$$
\begin{aligned}
& d\left(x_{i, j} y_{i, j}\right)=\left(y_{i, j}-\mu_{y}\right)\left(x_{i, j}-\mu_{x}\right) \\
& N D(X, Y)=\frac{1}{M N} \sum_{i=0}^{N} d\left(x_{i, j}, y_{i, j}\right)
\end{aligned}
$$

Difference of Pixel mean function between input image $X$ and output image $Y$ is denoted by $d\left(x_{i, j}, y_{i, j}\right)$. Normalized pixels intensity of $X$ and $Y$ on spatial location $(\mathrm{i}, \mathrm{j})$ is indicated by $x_{i, j}$ and $y_{i, j}$. With the help of beta distribution function and parameter $\alpha_{3}$ and $\beta_{3}$ the preservation of detail score function is defined as

$$
P D S=\frac{\Gamma\left(\alpha_{3}+\beta_{3}\right)}{\Gamma\left(\alpha_{3}\right) \Gamma\left(\beta_{3}\right)}(1-N D)^{\beta_{3}-1} N D^{\left(\alpha_{3}-1\right)}
$$

Where

$\Gamma($.$) is the Gamma function.$

$N P D S=\frac{P D S}{\operatorname{argmax}(P D S)}$

Here $N P D S$ is the normalized value of preservation of detail score function, which is the ratio of $P D S$ and max value of $P D S$. As relatively high and low detail preservation indicate low contrast enhancement and production of artifacts in US image respectively, so optimum value of preservation of detail will be obtained by using NPDS.

\subsection{Final Objective Function}

The construction of final objective function of MBORBHE highly depends on the characteristic of the image. For ultrasound image of knee joint cartilage the relation of every function to the expected ideal one should be examined. In ideal case, the mean value of brightness of input image and output image should be as close as to the peak value of NPBS. In case of enhancement of contrast the value should be as close as to the peak value of NOCS but should not be over enhanced. The feature of objects of input image should not be diminish or distorted in the output image will be considered by using the value of NPDS. The ideal case is, finding out the separating point that will satisfy the all above three conditions. But it is really impossible to satisfy three condition at the same time. For that reason the best solution is to find out the separating point that will best satisfy these criterions. By considering above three criteria the final objective function has been formulated as follows 
$N O b j(N P B S, N O C S, N P D S)=\frac{\alpha(N P B S)+\beta(N O C S)+\phi(N P D S)}{(\alpha+\beta+\phi)}$

(21)

We evaluated the performance of MBORBHE in case of ultrasound image of knee joint cartilage. For performance evaluation the proposed MBORBHE has been compared with the existing conventional HE, BBHE, DSIHE, RMSHE and MMBEBHE. We have used $r=4$ for all the recursive methods.

\section{Results}

SNR has been used for the measurement of brightness and contrast enhancement of the image. SNR is defined as

$$
S N R=10 \log _{10} \frac{\sum_{m=0}^{M-1} \sum_{n=0}^{N-1}[I(m, n)]^{2}}{M S E}
$$

Where,

$$
M S E=\frac{1}{M . N} \sum_{m=0}^{M-1} \sum_{n=0}^{N-1}\left[I(m, n)-I^{\prime}(m, n)\right]^{2}
$$

Here, $I$ is the original image, $I^{\prime}$ is the enhanced image of different contrast enhancement method compared for finding out the best one. SNR was then used to evaluate the standard of the output image quality. It was measured in decibels (dB). Hence, the higher the $S N R$ value, the better the resultant image quality. For the measurement of the detail preservation of the output image SSIM has been used. SSIM is defined as

$$
\operatorname{SSIM}(x, y)=\frac{\left(2 \mu_{x} \mu_{y}+C_{1}\right)\left(2 \sigma_{X Y}+C_{2}\right)}{\left(\mu_{x}^{2}+\mu_{y}^{2}+C_{1}\right)\left(\sigma_{x}^{2}+\sigma_{y}^{2}+C_{2}\right)}
$$

Here, $\sigma_{X Y}$ is the co-variance of $x$ and $y$. Entropy has been used for measurement of the contrast of the US image. It is defined as

$$
E n t[p]=-\sum_{k=0}^{L-1} p(k) \log _{2} p(k)
$$

Here, the probability density function (PDF) is $p . L$ is the number of gray level. Higher the value of entropy, larger contrast enhancement of the image. For the measurement of brightness preservation of the US images mean brightness difference has been used. For performance evaluation of the proposed method the output US image of different conventional HE methods and proposed method is shown in Figure1. The US image of knee joint cartilage has been collected from University Technology Malaysia (UTM), Johor Bahru, Malaysia. The name of the ultrasound (US) machine was 'aplio MX' and the company's name was TOSHIBA. We used 2D, 8MHz (PLT-805AT) linear probe for obtaining the US images. The quantitative values of different performance metric is shown in Table.1. Figure 2 and 3 represent Entropy and mean brightness of output image of different HE methods.

Table I: Numerical values of different performance metrics for ultrasound image of cartilage

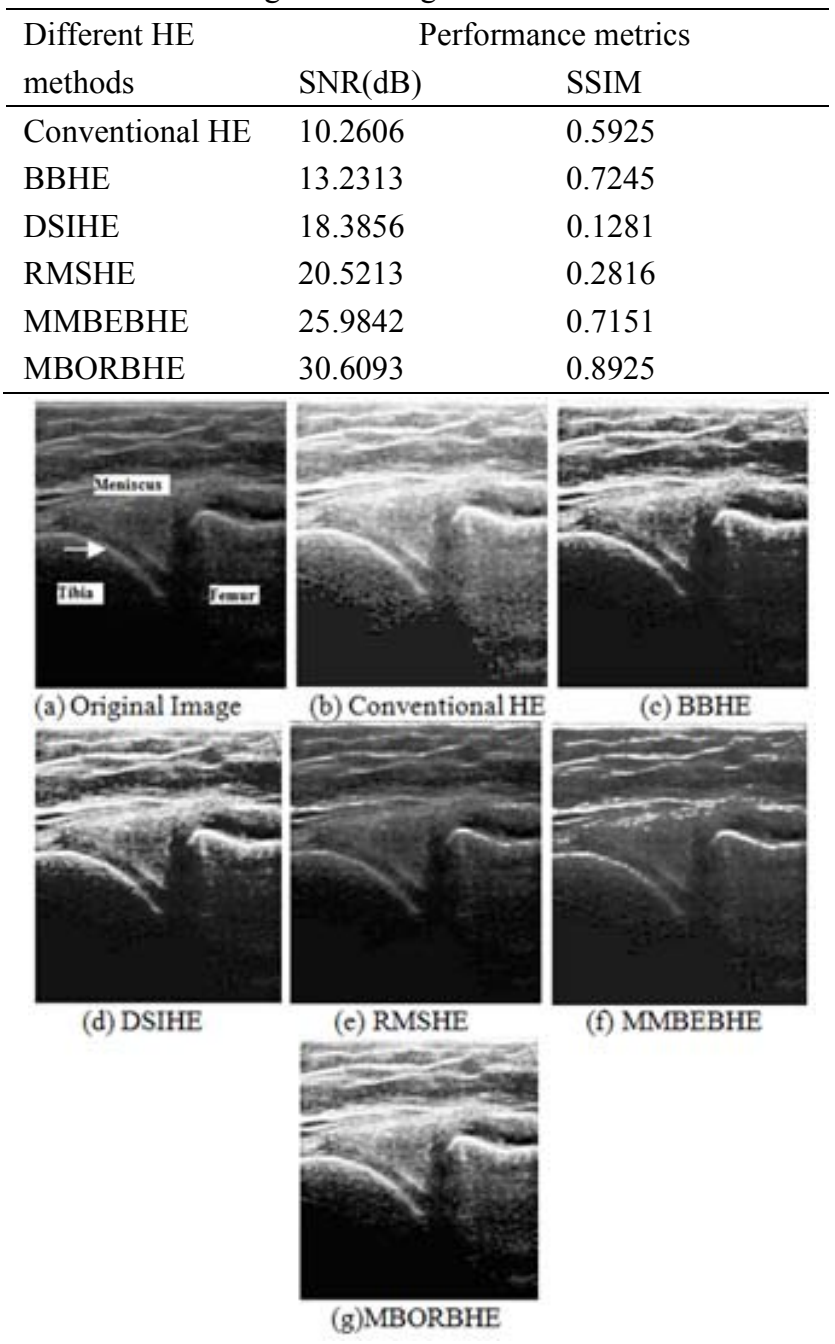

Fig.1.(a) Original Cartilage Image (b) Conventional HE (c) BBHE (d) DSIHE (e) RMSHE (f) MMBEBHE (g) MBORBHE (proposed)

For the qualitative analysis of the proposed MBORBHE method the original image of knee joint cartilage (Medial position) is shown in Figure 1(a) and the arrow of Figure 1(a) indicates ' $V$ ' shape cartilages layer of knee joints. The enhancement of the original image by using conventional HE, BBHE, DSIHE, RMSHE, MMBEBHE and proposed MBORBHE are shown in Figure 1(b), 1(c), 1(d), 1(e), 1(f) and 1(g) respectively. The resultant images from the conventional $\mathrm{HE}, \mathrm{BBHE}$ and DSIHE (shown in Figure 1(b), 1(c) and 1(d)) had larger mean brightness which were much brighter compared to the 
original image, therefore they resulted in unpleasant contrast enhancement. From Figure 1(b), it can be observed the unnatural brightness enhancement, not only in meniscus and cartilage layer, but also in the upper portion of the tibia and femur of the knee joint. Though unnatural brightness enhancement was not so significant in case of Figure 1(c), some detail loss artifacts are observed between the joint of tibia and lower part of the ' $V$ ' shape cartilage. Unnatural brightness enhancement was also noticeable in case of Figure 1(d). In case of RMSHE and MMBEBHE in Figure 1(e) and 1(f), it could be seen the obvious change in brightness (darker comparing with other enhancement methods) which decreased the contrast around the area of the layer of cartilage. The output images of these two methods also give less emphasis on the important details preservation. From Figure 1(e), it is observed the detail loss artifact in the junction of lower part of ' $\mathrm{V}$ ' shape cartilage and tibia of the knee. In case of Figure 1(f), the detail loss artifacts was also observable in the border of ' $\mathrm{V}$ ' shaped cartilage. However, in case of Figure $1(\mathrm{~g})$ this kind of detail loss artifacts is not observed very actively. It could be see a natural enhancement of the shape of cartilage. The ' $\mathrm{V}$ ' shape of the cartilage was clearly noticeable, as shown in Figure $1(\mathrm{~g})$. The shape of the Meniscus collateral ligament was also clear compared with other methods. In case of Figure 1(b), 1(c), 1(d), 1(e) and 1(f), it is seen some unnatural white lines at the border of the cartilage layer. It was also observed that the white border of the cartilage generated by the MBORBHE was natural.

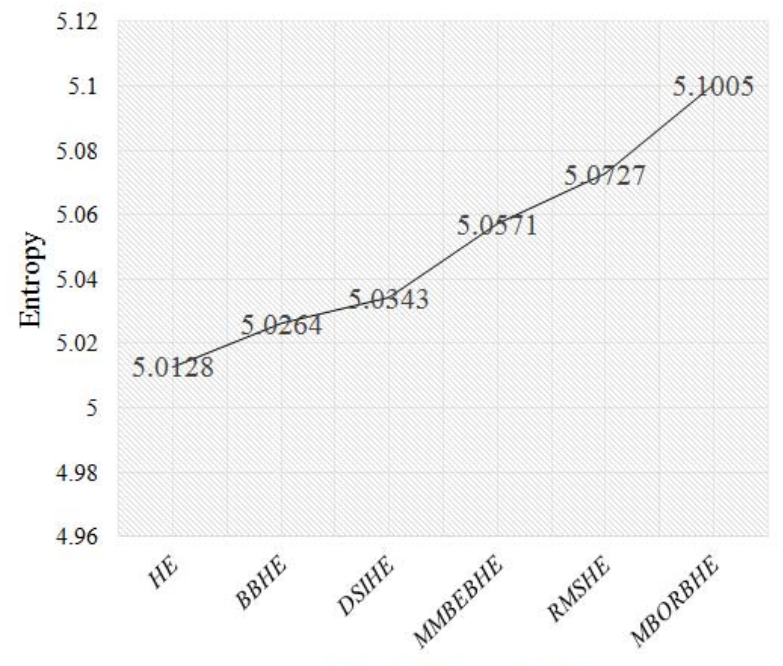

Different HE methods

Fig 2. Entropy of output image for different HE methods

Figure 2 shows the values of entropy of output image for different HE methods. From the graphical presentation it was clear that the entropy for the proposed method was higher than other methods. That's mean the contrast enhancement of the output image for the proposed method was much better than other methods.

Figure 3 shows mean brightness of the output image for different HE methods. From this bar graph it was clear that the brightness of the original image and the output image for the proposed method were almost same. Where for RMSHE and MMBEBHE the brightness has been decreased and for other methods the brightness has been increased. So we can say that brightness has been successfully preserved in case of the proposed method.

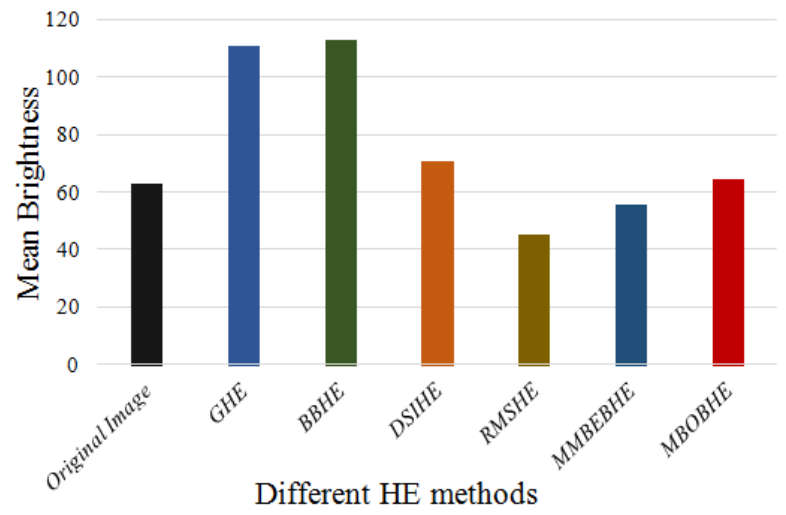

Fig.3 Mean Brightness of resultant image for different HE methods

\section{Conclusion}

From the qualitative and quantitative analysis it was clear that the proposed method outperform other conventional HE methods. It was possible to enhance the contrast of the US image with preservation of brightness and important details by using the proposed MBORBHE method. From the obtained qualitative and quantitative results it can be said that optimum separating point for histogram segmentation of image had been obtained successfully by considering three objective functions. From the result a conclusion can be drawn that, the proposed method had successfully removed the limitation of low contrast of US image. It will be helpful for detecting early knee OA by filtering US image of knee joint cartilage. In future optimum edge preservation function will be included for further development of the proposed method.

\section{Acknowledgment}

This research received funding from University 
Malaya Research Grant; UMRG (RP020A-13AET), University of Malaya.

\section{References}

(1) C.F. Eckstein F, Raynauld J, Waterton JC, Peterfy C., 'Magnetic resonance imaging (MRI) of articular cartilage in knee osteoarthritis (OA): morphological assessment.', Osteoarthr Cartil 2006;, 2006, 14 (Suppl A),pp. 46-75.

(2) R.C. Gonzalez, Woods, R.E, 'Digital Image Processing', Prentice-Hall, New Jersey.,2002.

(3) J. Du, Fain, S.B., Gu, T.L., Grist, T.M., Mistretta, C.A.,, 'Noise reduction in MR angiography with nonlinear anisotropic filtering', Journal of Magnetic Resonance Imaging,2004,19(5),pp. 632-639.

(4) A. John, Huda, W., Scalzetti, E.M., Ogden, K.M., Roskopf, M.L., 'Performance of a single look up table (LUT) for displaying chest CT images', Academic Radiology,2004,11(6),pp. 609-616.

(5) M. Weichselbaum, Sparrow, M.P., Hamilton, E.J., Thompson, P.J., Knight, D.A., 'A confocal microscopic study of solitary pulmonary neuroendocrine cells in human airway epithelium', Respiratory Research,2005,6(115),pp. 1-11.

(6) D.S. Bright, 'MacLispix: a special purpose public domain image analysis program for the Macintosh.', Microbeam Analysis,1995,4,pp. 151-163.

(7) W.S. Rasband, Bright, D.S.,, 'NIH Image: a public domain image processing program for the Macintosh.', Microbeam Analysis Society Journal 1995,4,pp. $137-149$

(8) Y. Kim, 'Contrast enhancement using brightness preserving bihistogram equalization', IEEE Transactions on Consumer Electronics, , 1997, 43(1),pp. 1-8.

(9) Y. Wang, Q. Chen, and B. Zhang, 'Image enhancement based on equal area dualistic sub-image histogram equalization method', Consumer Electronics, IEEE Transactions on, 1999, 45(1),pp. 68-75.

(10) S.-D. Chen and A.R. Ramli, 'Contrast enhancement using recursive mean-separate histogram equalization for scalable brightness preservation', Consumer Electronics, IEEE Transactions on,2003,49(4),pp. 1301-1309.

(11)K. Sim, C. Tso, and Y. Tan, 'Recursive sub-image histogram equalization applied to gray scale images', Pattern Recognition Letters,2007,28(10),pp.
1209-1221.

(12) S.-D. Chen and A.R. Ramli, 'Minimum mean brightness error bi-histogram equalization in contrast enhancement', Consumer Electronics, IEEE Transactions on,2003,49(4),pp. 1310-1319. 